\title{
Local existence and uniqueness of solutions of a degenerate parabolic system
}

\author{
Dazhi Zhang, Jiebao Sun ${ }^{*}$ and Boying Wu
}

* Correspondence: sunjiebao@126. com

Department of Mathematics, Harbin Institute of Technology, Harbin 150001, PR China,

\begin{abstract}
This article deals with a degenerate parabolic system coupled with general nonlinear terms. Using the method of regularization and monotone iteration technique, we obtain the local existence of solutions to the Dirichlet initial boundary value problem. We also establish the uniqueness of the solution if the reaction terms satisfy the Lipschitz condition.
\end{abstract}

Keywords: Existence, Uniqueness, Degenerate, Monotone iteration

\section{Introduction}

In this article, we consider the following degenerate parabolic system

$$
\begin{array}{lr}
\frac{\partial u_{i}}{\partial t}=\Delta u_{i}^{m_{i}}+f_{i}\left(x, t, u_{1}, u_{2}\right), & (x, t) \in Q_{T}, \\
u_{i}(x, t)=0, & (x, t) \in \partial \Omega \times(0, T), \\
u_{i}(x, 0)=u_{i 0}(x), & x \in \Omega,
\end{array}
$$

where $m_{i}>1, i=1,2, Q_{T}=\Omega \times(0, T), \Omega$ is a bounded domain in $\mathbb{R}^{N}$ with smooth boundary, $f_{i}\left(x, t, u_{1}, u_{2}\right) \in C\left(\bar{\Omega} \times[0, T] \times \mathbb{R}^{2}\right)$ and $0 \leq u_{i 0} \in L^{\infty}(\Omega) \cap H_{0}^{1}(\Omega)$.

The coupled equations in (1.1) provide a class of quasilinear degenerate parabolic systems. Problems of this form arise in a number of areas of science. For instance, in models for gas or fluid flow in porous media [1-3] and for the spread of certain biological populations [4-6]. When $m_{1}=m_{2}=1$, the system (1.1) models the Newtonian fluids, which is couples with Laplace equations. For various initial boundary problems to this kind system, many articles have been devoted to the existence of the solutions and blowup properties of the solutions [7-9].

In recent years, degenerate parabolic systems are of particular interests since they can take into account nonlinear diffusion occurring in the phenomena appearing in the models, and have been extensively studied by many researchers (see e.g., [3,10-13] and the references therein). The degeneracy and coupled with nonlinear terms of this systems cause great difficulties to study them. In this article, we will establish the local existence and uniqueness results under some special cases for the nonlinear reaction terms. First, by making use the method of regularization and monotone iteration technique, we obtain a sequence of approximation solutions. Then a weak solution is

\section{SpringerOpen ${ }^{\circ}$}

(C) 2011 Zhang et al; licensee Springer. This is an Open Access article distributed under the terms of the Creative Commons Attribution License (http://creativecommons.org/licenses/by/2.0), which permits unrestricted use, distribution, and reproduction in any medium, provided the original work is properly cited. 
obtained as the limit of the solutions of such problems. Executing this program one encounters two difficulties. The first is proving that the approximating problems which are nondegenerate admits a solution, the second difficulty is to establish uniform estimates for these solutions. At last, we establish the uniqueness results when the reaction terms satisfy the Lipschitz condition.

Since the system (1.1) is degenerate whenever $u_{1}, u_{2}$ vanish, there is no classical solution in general. So we focus our main efforts on the discussion of weak solutions in the sense of the following.

Definition 1.1. A nonnegative vector-valued function $u=\left(u_{1}, u_{2}\right)$ is called to be a weak solution of the problem (1.1)-(1.3) provided that $u_{i}^{m_{i}} \in L^{2}\left(0, T ; H_{0}^{1}(\Omega)\right) \cap L^{\infty}\left(Q_{T}\right)$, $\partial u_{i}^{m_{i}} / \partial t \in L^{2}\left(Q_{T}\right)$, and

$$
\iint_{Q_{T}}-u_{i} \frac{\partial \varphi_{i}}{\partial t}+\nabla u_{i}^{m_{i}} \nabla \varphi_{i} \mathrm{~d} x \mathrm{~d} t-\int_{\Omega} u_{i 0}(x) \varphi_{i}(x, 0) \mathrm{d} x=\iint_{Q_{T}} f_{i}\left(x, t, u_{1}, u_{2}\right) \varphi_{i} \mathrm{~d} x \mathrm{~d} t,
$$

for any test function $\varphi_{i} \in C^{2}\left(\bar{Q}_{T}\right)$ with $\left.\phi_{i}\right|_{\partial \Omega \times(0, T)}=0, \phi_{i}(x, T)=0, i=1,2$. The above equation also implies

$$
\begin{gathered}
\int_{0}^{t} \int_{\Omega}-u_{i} \frac{\partial \varphi_{i}}{\partial t}+\nabla u_{i}^{m_{i}} \nabla \varphi_{i} \mathrm{~d} x \mathrm{~d} t+\int_{\Omega} u_{i}(x, t) \varphi_{i}(x, t) \mathrm{d} x-\int_{\Omega} u_{i 0}(x) \varphi_{i}(x, 0) \mathrm{d} x \\
=\int_{0}^{t} \int_{\Omega} f_{i}\left(x, t, u_{1}, u_{2}\right) \varphi_{i} \mathrm{~d} x \mathrm{~d} t, \quad \text { a.e. } \quad t \in(0, T) .
\end{gathered}
$$

Definition 1.2. A function $f=f\left(u_{1}, u_{2}\right)$ is said to be quasimonotone nondecreasing (respectively, nonincreasing) if for fixed $u_{1}$ (or $u_{2}$ ), $f$ is nondecreasing (respectively, nonincreasing) in $u_{2}$ (or $u_{1}$ ).

Throughout this article, we assume $f_{i}\left(x, t, u_{1}, u_{2}\right)(i=1,2)$ satisfies the following condition:

(A0) $f_{i}\left(x, t, u_{1}, u_{2}\right)(i=1,2)$ is quasimonotonically nondecreasing for $u_{1}, u_{2}$.

(A1) There exists a nonnegative function $g(u) \in C^{1}(\mathbb{R})$ such that

$$
\left|f_{i}\left(x, t, u_{1}, u_{2}\right)\right| \leq \min \left\{g\left(u_{1}\right), g\left(u_{2}\right)\right\} \quad \text { for all }(x, t) \in Q_{T}, u_{1}, u_{2} \in \mathbb{R} \text {. }
$$

\section{Existence and uniqueness}

In this section, we show the local existence and uniqueness of weak solutions of (1.1)(1.3). First, we show the local existence results.

Theorem 2.1. Assume (AO), (A1) hold, then there exists a constant $T_{1} \in[0, T]$ such that (1.1)-(1.3) admits a solution $\left(u_{1}, u_{2}\right)$ in $Q_{T_{1}}$.

Proof. Due to the degeneracy of the system (1.1), we consider the following regularized problem

$$
\begin{aligned}
& \frac{\partial u_{i}}{\partial t}=\operatorname{div}\left(\left(m_{i} u_{i}^{m_{1}-1}+\varepsilon\right) \nabla u_{i}\right)+f_{i \varepsilon}\left(x, t, u_{1}, u_{2}\right), \quad(x, t) \in Q_{T} \\
& u_{i}(x, t)=0, \quad(x, t) \in \partial \Omega \times(0, T),
\end{aligned}
$$




$$
u_{i}(x, 0)=u_{i 0 \varepsilon}(x), \quad x \in \Omega,
$$

where $f_{i \varepsilon} \in C^{1}\left(\bar{\Omega} \times[0, T] \times \mathbb{R}^{2}\right) ; f_{i \varepsilon} \rightarrow f_{i}$ uniformly on bounded subsets of $\bar{\Omega} \times[0, T] \times \mathbb{R}^{2}$, and $f_{i \varepsilon}$ satisfies the assumptions (A0), (A1), $u_{i 0 \varepsilon}(x) \in C_{0}^{\infty}(\Omega)$, $u_{i 0 \varepsilon}^{m_{i}} \rightarrow u_{i 0}^{m_{i}}, u_{i 0 \varepsilon}^{m_{i}} \rightarrow u_{i 0}^{m_{i}}$, strongly in $W_{0}^{1,2}(\Omega)$ as $\varepsilon \rightarrow 0$.

Now we will prove that the regularized problem (2.1)-(2.3) admits a classical solution. Construct a sequence $\left\{\left(u_{1 \varepsilon}^{(k)}, u_{2 \varepsilon}^{(k)}\right)\right\}_{k=1}^{\infty}$ from the following iteration process

$$
\begin{aligned}
& \frac{\partial u_{i}^{(k)}}{\partial t}-\operatorname{div}\left(\left(m_{i}\left(u_{i}^{(k)}\right)^{m_{i}-1}+\varepsilon\right) \nabla u_{i}^{(k)}\right)=f_{i \varepsilon}\left(x, t, u_{1 \varepsilon}^{(k-1)}, u_{2 \varepsilon}^{(k-1)}\right), \quad(x, t) \in Q_{T}, \\
& u_{i \varepsilon}^{(k)}(x, t)=0, \\
& (x, t) \in \partial \Omega \times(0, T), \\
& u_{i 0 \varepsilon}^{(k)}(x, 0)=u_{i 0 \varepsilon}(x), \quad x \in \Omega,
\end{aligned}
$$

with a suitable initial value $\left(u_{1 \varepsilon}^{(0)}, u_{2 \varepsilon}^{(0)}\right), i=1,2$. By classical results in [14], the problem (2.4)-(2.6) admits a classical solution $\left(u_{1 \varepsilon}^{(k)}, u_{2 \varepsilon}^{(k)}\right)$ for fixed $k$ and $\varepsilon$ when $\left(u_{1 \varepsilon}^{(k-1)}, u_{2 \varepsilon}^{(k-1)}\right)$ is smooth. The choice of the initial iteration value which will be obtained by the quasimonotone property of $\left(f_{1}, f_{2}\right)$ would be crucial to ensure that the above sequence converges to a solution of the generalized problem.

Let $\left(\underline{u}_{1 \varepsilon}^{(0)}(x, t), \underline{u}_{2 \varepsilon}^{(0)}(x, t)\right)=\left(\inf _{\Omega}\left\{u_{10 \varepsilon}(x)\right\}, \inf _{\Omega}\left\{u_{20 \varepsilon}(x)\right\}\right)$, and $\left(\underline{u}_{1 \varepsilon}^{(1)}, \underline{u}_{2 \varepsilon}^{(1)}\right)$ be a classical solution of the following problem

$$
\begin{array}{ll}
\frac{\partial \underline{u}_{i}^{(1)}}{{ }_{i}}-\operatorname{div}\left(\left(m_{i}\left(\underline{u}_{i}^{(1)}\right)^{m_{i}-1}+\varepsilon\right) \nabla \underline{u}_{i}^{(1)}\right)=f_{i \varepsilon}\left(x, t, \underline{u}_{1 \varepsilon}^{(0)}, \underline{u}^{(0)}\right), & (x, t) \in Q_{T}, \\
{ }^{\partial t}{ }^{(1)}(x, t)=0, & (x, t) \in \partial \Omega \times(0, T), \\
\underline{i}_{i \varepsilon} \underline{u}_{i 0 \varepsilon}^{(1)}(x, 0)=u_{i 0 \varepsilon}(x) \geq \underline{u}_{i \varepsilon}^{(0)}(x), & x \in \Omega .
\end{array}
$$

By the comparison theorem [15], we have

$$
\underline{u}_{1 \varepsilon}^{(1)} \geq \underline{u}_{1 \varepsilon}^{(0)}, \underline{u}_{2 \varepsilon}^{(1)} \geq \underline{u}_{2 \varepsilon}^{(0)} .
$$

Then the quasimonotone nondecreasing property of $f_{i \varepsilon}$ shows that

$$
\begin{aligned}
& f_{1 \varepsilon}\left(x, t, \underline{u}_{1 \varepsilon}^{(1)}, \underline{u}_{2 \varepsilon}^{(1)}\right) \geq f_{1 \varepsilon}\left(x, t, \underline{u}_{1 \varepsilon}^{(0)}, \underline{u}_{2 \varepsilon}^{(1)}\right) \geq f_{1 \varepsilon}\left(x, t, \underline{u}_{1 \varepsilon}^{(0)}, \underline{u}_{2 \varepsilon}^{(0)}\right), \\
& f_{2 \varepsilon}\left(x, t, \underline{u}_{1 \varepsilon}^{(1)}, \underline{u}_{2 \varepsilon}^{(1)}\right) \geq f_{2 \varepsilon}\left(x, t, \underline{u}_{1 \varepsilon}^{(1)}, \underline{u}_{2 \varepsilon}^{(0)}\right) \geq f_{2 \varepsilon}\left(x, t, \underline{u}_{1 \varepsilon}^{(0)}, \underline{u}_{2 \varepsilon}^{(0)}\right) .
\end{aligned}
$$

Then we can also obtain a classical solution $\left(\underline{u}_{1 \varepsilon}^{(2)}, \underline{u}_{2 \varepsilon}^{(2)}\right)$ from $(2.4)-(2.6)$ when $k=2$, and $\underline{u}_{1 \varepsilon}^{(2)} \geq \underline{u}_{1 \varepsilon}^{(1)}, \underline{u}_{2 \varepsilon}^{(2)} \geq \underline{u}_{2 \varepsilon}^{(1)}$. So we can obtain a nondecreasing sequence

$$
\underline{u}_{i \varepsilon}^{(0)} \leq \underline{u}_{i \varepsilon}^{(1)} \leq \underline{u}_{i \varepsilon}^{(2)} \leq \cdots \leq \underline{u}_{i \varepsilon}^{(k)} \leq \cdots
$$

With the similar method, by setting $\left(\bar{u}_{1 \varepsilon}^{(0)}(x, t), \bar{u}_{2 \varepsilon}^{(0)}(x, t)\right)=\left(\sup _{Q_{T}}\left\{u_{10 \varepsilon}(x)\right\}, \sup _{Q_{T}}\left\{u_{20 \varepsilon}(x)\right\}\right)$, we obtain a classical solution $\left(\bar{u}_{1 \varepsilon}^{(1)}, \bar{u}_{2 \varepsilon}^{(1)}\right)$ of the following problem 


$$
\begin{array}{ll}
\frac{\partial \bar{u}_{i}^{(1)}}{\partial t}-\operatorname{div}\left(\left(m_{i}\left(\bar{u}_{i}^{(1)}\right)^{m_{i}-1}+\varepsilon\right) \nabla \bar{u}_{i}^{(1)}\right)=f_{i \varepsilon}\left(x, t, \bar{u}_{1 \varepsilon}^{(0)}, \bar{u}_{2 \varepsilon}^{(0)}\right), & (x, t) \in Q_{T}, \\
\bar{u}_{i \varepsilon}^{(1)}(x, t)=0, & (x, t) \in \partial \Omega \times \\
\bar{u}_{i 0 \varepsilon}^{(1)}(x, 0)=u_{i 0 \varepsilon}(x) \leq \bar{u}_{i \varepsilon}^{(0)}(x), & x \in \Omega,
\end{array}
$$

and

$$
\bar{u}_{1 \varepsilon}^{(1)} \leq \bar{u}_{1 \varepsilon}^{(0)}, \bar{u}_{2 \varepsilon}^{(1)} \leq \bar{u}_{2 \varepsilon}^{(0)} .
$$

And the quasimonotone nondecreasing property of $f_{i \varepsilon}$ also shows that

$$
\bar{u}_{i \varepsilon}^{(0)} \geq \bar{u}_{i \varepsilon}^{(1)} \geq \bar{u}_{i \varepsilon}^{(2)} \geq \cdots \geq \bar{u}_{i \varepsilon}^{(k)} \geq \cdots .
$$

Now we show

$$
\underline{u}_{i \varepsilon}^{(0)} \leq \underline{u}_{i \varepsilon}^{(1)} \leq \underline{u}_{i \varepsilon}^{(2)} \leq \cdots \leq \underline{u}_{i \varepsilon}^{(k)} \leq \underline{u}_{i \varepsilon}^{(k+1)} \leq \bar{u}_{i \varepsilon}^{(k+1)} \leq \bar{u}_{i \varepsilon}^{(k)} \leq \cdots \leq \bar{u}_{i \varepsilon}^{(2)} \leq \bar{u}_{i \varepsilon}^{(1)} \leq \bar{u}_{i \varepsilon}^{(0)}
$$

It is obvious that $\underline{u}_{i \varepsilon}^{(0)} \leq \bar{u}_{i \varepsilon}^{(0)}$. Assume that $\underline{u}_{i \varepsilon}^{(k)} \leq \underline{u}_{i \varepsilon}^{(k)}$, we just need to prove that $\underline{u}_{i \varepsilon}^{(k+1)} \leq \underline{u}_{i \varepsilon}^{(k+1)}$. Since $f_{i \varepsilon}$ is quasimonotone nondecreasing, we have

$$
\begin{aligned}
& f_{1 \varepsilon}\left(x, t, \underline{u}_{1 \varepsilon}^{(k)}, \underline{u}_{2 \varepsilon}^{(k)}\right) \leq f_{1 \varepsilon}\left(x, t, \bar{u}_{1 \varepsilon}^{(k)}, \underline{u}_{2 \varepsilon}^{(k)}\right) \leq f_{1 \varepsilon}\left(x, t, \bar{u}_{1 \varepsilon}^{(k)}, \bar{u}_{2 \varepsilon}^{(k)}\right), \\
& f_{2 \varepsilon}\left(x, t, \underline{u}_{1 \varepsilon}^{(k)}, \underline{u}_{2 \varepsilon}^{(k)}\right) \leq f_{2 \varepsilon}\left(x, t, \underline{u}_{1 \varepsilon}^{(k)}, \bar{u}_{2 \varepsilon}^{(k)}\right) \leq f_{2 \varepsilon}\left(x, t, \bar{u}_{1 \varepsilon}^{(k)}, \bar{u}_{2 \varepsilon}^{(k)}\right) .
\end{aligned}
$$

From the iteration equations

$$
\begin{array}{ll}
\frac{\partial \underline{u}_{i}^{(k+1)}}{\partial t}-\operatorname{div}\left(\left(m_{i}\left(\underline{u}_{i}^{(k+1)}\right)^{m_{1}-1}+\varepsilon\right) \nabla \underline{u}_{i}^{(k+1)}\right)=f_{i \varepsilon}\left(x, t, \underline{u}_{1 \varepsilon}^{(k)}, \underline{u}_{2 \varepsilon}^{(k)}\right),(x, t) \in Q_{T}, & \\
\frac{\partial \bar{u}_{i}^{(k+1)}}{\partial t}-\operatorname{div}\left(\left(m_{i}\left(\bar{u}_{i}^{(k+1)}\right)^{m_{1}-1}+\varepsilon\right) \nabla \bar{u}_{i}^{(k+1)}\right)=f_{i \varepsilon}\left(x, t, \bar{u}_{1 \varepsilon}^{(k)}, \bar{u}_{2 \varepsilon}^{(k)}\right), & (x, t) \in Q_{T}, \\
\underline{u}_{i \varepsilon}^{(k+1)}(x, t)=0=\bar{u}_{i \varepsilon}^{(k+1)}(x, t), & (x, t) \in \partial \Omega \times(0, T), \\
\underline{u}_{i 0 \varepsilon}^{(k+1)}(x, 0)=u_{i 0 \varepsilon}(x)=\bar{u}_{i \varepsilon}^{(k+1)}(x, 0), & x \in \Omega,
\end{array}
$$

and the comparison theorem, we have $\underline{u}_{i \varepsilon}^{(k+1)} \leq \underline{u}_{i \varepsilon}^{(k+1)}$. Further we can obtain (2.7).

Let $\left(u_{1 \varepsilon}^{(k)}, u_{2 \varepsilon}^{(k)}\right)=\left(\underline{u}_{1 \varepsilon}^{(k)}, \underline{u}_{2 \varepsilon}^{(k)}\right)$, then $\left\{\left(u_{1 \varepsilon}^{(k)}, u_{2 \varepsilon}^{(k)}\right)\right\}_{k=1}^{\infty}$ is a nondecreasing bounded sequence.

Then there exist functions $u_{i \varepsilon}(i=1,2)$ such that

$$
\lim _{k \rightarrow \infty} u_{i \varepsilon}^{(k)}=u_{i \varepsilon}, \quad \text { a.e. } \quad \text { in } \quad Q_{T} .
$$

The continuity of function $f_{i \varepsilon}(i=1,2)$ also shows that

$$
\lim _{k \rightarrow \infty} f_{i \varepsilon}\left(x, t, u_{1 \varepsilon}^{(k)}, u_{2 \varepsilon}^{(k)}\right)=f_{i \varepsilon}\left(x, t, u_{1 \varepsilon}, u_{2 \varepsilon}\right) \text {, a.e. in } Q_{T} .
$$

Therefore, we claim that there exist $T_{1} \in(0, T]$ and a positive constant $M$ (independent of $\varepsilon$ and $k$ ), such that for all $k$,

$$
\left|u_{i \varepsilon}^{(k)}\right|_{L^{\infty}\left(Q_{T_{1}}\right)} \leq M, \quad i=1,2 .
$$


Let $v_{i}^{ \pm}(t)$ be the solutions of the ordinary differential equations

$$
\frac{\mathrm{d} v_{i}^{ \pm}(t)}{\mathrm{d} t}= \pm g\left(v_{i}\right), \quad v_{i}^{ \pm}(0)= \pm\left|u_{i 0}\right|_{L^{\infty}(\Omega)}, \quad i=1,2 .
$$

The results in [16] show that there exists $T_{i}^{*} \in(0, T), i=1,2$, such that $v_{i}^{ \pm}(t)$ exists on $\left[0, T_{i}^{*}\right]$ with $T_{i}^{*}$ depends only on $\left|u_{i 0}\right|_{L^{\infty}(\Omega)}$. By the comparison theorem, we have

$$
\left|u_{i \varepsilon}^{(k)}(x, t)\right| \leq \max \left\{v_{i}^{+}(t),-v_{i}^{-}(t)\right\}, \quad i=1,2 .
$$

Then by setting $T_{1}=\frac{1}{2} \min \left\{T_{1}^{*}, T_{2}^{*}\right\}$ and $M=\max \left\{v_{i}^{+}\left(T_{1}\right),-v_{i}^{-}\left(T_{1}\right)\right\}$, we obtain (2.10).

Now we show that $\left(u_{i \varepsilon}^{(k)}\right)^{m_{i}}+\varepsilon u_{i \varepsilon}^{(k)} \rightarrow u_{i \varepsilon}^{m_{i}}+\varepsilon u_{i \varepsilon} \quad$ in $L^{2}\left(0, T_{1} ; H_{0}^{1}(\Omega)\right)$, $\left(u_{i \varepsilon}^{(k)}\right)_{t}^{m_{i}} \rightarrow\left(u_{i \varepsilon}^{m_{i}}\right)_{t}, u_{i \varepsilon t}^{(k)} \rightarrow u_{i \varepsilon t}$ in $L^{2}\left(Q_{T_{1}}\right)$ as $k \rightarrow \infty$, where $\rightarrow$ stands for weak convergence.

Multiplying (2.4) by $\left(u_{i \varepsilon}^{(k)}\right)^{m_{i}}+\varepsilon u_{i \varepsilon}^{(k)}$ and integrating over $Q_{T_{1}}=\Omega \times\left(0, T_{1}\right)$, we have

$$
\begin{array}{r}
\iint_{Q_{T_{1}}}\left[\left(u_{i \varepsilon}^{(k)}\right)^{m_{i}}+\varepsilon u_{i \varepsilon}^{(k)}\right] \frac{\partial u_{i \varepsilon}^{(k)}}{\partial t} \mathrm{~d} t \mathrm{~d} x+\iint_{\mathrm{Q}_{1}}\left|\nabla\left(u_{i \varepsilon}^{(k)}\right)^{m_{i}}+\varepsilon \nabla u_{i \varepsilon}^{(k)}\right|^{2} \mathrm{~d} x \mathrm{~d} t \\
=\iint_{\mathrm{Q}_{1}} f_{i \varepsilon}\left(x, t, u_{1 \varepsilon}^{(k-1)}, u_{2 \varepsilon}^{(k-1)}\right)\left[\left(u_{i \varepsilon}^{(k)}\right)^{m_{i}}+\varepsilon u_{i \varepsilon}^{(k)}\right] \mathrm{d} x \mathrm{~d} t,
\end{array}
$$

that is

$$
\begin{aligned}
& \iint_{Q_{T_{1}}}\left|\nabla\left(u_{i \varepsilon}^{(k)}\right)^{m_{i}}+\varepsilon \nabla u_{i \varepsilon}^{(k)}\right|^{2} \mathrm{~d} x \mathrm{~d} t \\
&=\iint_{Q_{T_{1}}} f_{i \varepsilon}\left(x, t, u_{1 \varepsilon}^{(k-1)}, u_{2 \varepsilon}^{(k-1)}\right)\left[\left(u_{i \varepsilon}^{(k)}\right)^{m_{i}}+\varepsilon u_{i \varepsilon}^{(k)}\right] \mathrm{d} x \mathrm{~d} t \\
& \quad-\frac{1}{m_{i}+1} \int_{\Omega}\left[\left(u_{i \varepsilon}^{(k)}\left(x, T_{1}\right)\right)^{m_{i}+1}-\left(u_{i \varepsilon}^{(k)}(x, 0)\right)^{m_{i}+1}\right] \mathrm{d} x \\
& \quad-\frac{1}{2} \int_{\Omega}\left[\left(u_{i \varepsilon}^{(k)}\left(x, T_{1}\right)\right)^{2}-\left(u_{i \varepsilon}^{(k)}(x, 0)\right)^{2}\right] \mathrm{d} x .
\end{aligned}
$$

Then by (2.10) and the property of $f_{i \varepsilon}$, we have

$$
\iint_{\mathrm{Q}_{1}}\left|\nabla\left(u_{i \varepsilon}^{(k)}\right)^{m_{i}}+\varepsilon \nabla u_{i \varepsilon}^{(k)}\right|^{2} \mathrm{~d} x \mathrm{~d} t \leq C,
$$

where $\mathrm{C}$ is a constant independent of $k, \varepsilon$.

Multiplying (2.4) by $\frac{\partial}{\partial t}\left[\left(u_{i \varepsilon}^{(k)}\right)^{m_{i}}+\varepsilon u_{i \varepsilon}^{(k)}\right]$ and integrating over $Q_{T_{1}}$, by Young's inequality we have 


$$
\begin{aligned}
& \iint_{Q_{T_{1}}} \frac{\partial u_{i \varepsilon}^{(k)}}{\partial t} \frac{\partial\left(u_{i \varepsilon}^{(k)}\right)^{m_{i}}}{\partial t} \mathrm{~d} x \mathrm{~d} t+\varepsilon \iint_{Q_{T_{1}}}\left|\frac{\partial u_{i \varepsilon}^{(k)}}{\partial t}\right|^{2} \mathrm{~d} x \mathrm{~d} t \\
& =-\frac{1}{2} \int_{0}^{T_{1}} \frac{\partial}{\partial t} \int_{\Omega}\left|\nabla\left(u_{i \varepsilon}^{(k)}\right)^{m_{i}}+\varepsilon \nabla u_{i \varepsilon}^{(k)}\right|^{2} \mathrm{~d} x \mathrm{~d} t+\varepsilon \iint_{\mathrm{Q}_{1}} f_{i \varepsilon}\left(x, t, u_{1 \varepsilon}^{(k-1)}, u_{2 \varepsilon}^{(k-1)}\right) \frac{\partial u_{i \varepsilon}^{(k)}}{\partial t} \mathrm{~d} x \mathrm{~d} t \\
& +\iint_{Q_{T_{1}}} f_{i \varepsilon}\left(x, t, u_{1 \varepsilon}^{(k-1)}, u_{2 \varepsilon}^{(k-1)}\right) \frac{\partial\left(u_{i \varepsilon}^{(k)}\right)^{m_{i}}}{\partial t} \mathrm{~d} x \mathrm{~d} t \\
& =-\frac{1}{2} \int_{0}^{T_{1}} \frac{\partial}{\partial t} \int_{\Omega}\left|\nabla\left(u_{i \varepsilon}^{(k)}\right)^{m_{i}}+\varepsilon \nabla u_{i \varepsilon}^{(k)}\right|^{2} \mathrm{~d} x \mathrm{~d} t+\varepsilon \iint_{Q_{T_{1}}} f_{i \varepsilon}\left(x, t, u_{1 \varepsilon}^{(k-1)}, u_{2 \varepsilon}^{(k-1)}\right) \frac{\partial u_{i \varepsilon}^{(k)}}{\partial t} \mathrm{~d} x \mathrm{~d} t \\
& +\frac{2 m_{i}}{m_{i}+1} \iint_{Q_{T_{1}}} f_{i \varepsilon}\left(x, t, u_{1 \varepsilon}^{(k-1)}, u_{2 \varepsilon}^{(k-1)}\right)\left(u_{i \varepsilon}^{(k)}\right)^{\left(m_{i}-1\right) / 2} \frac{\partial\left(u_{i \varepsilon}^{(k)}\right)^{\left(m_{i}+1\right) / 2}}{\partial t} \mathrm{~d} x \mathrm{~d} t \\
& \leq \frac{1}{2} \int_{\Omega}\left|\nabla\left(u_{i 0 \varepsilon}^{(k)}\right)^{m_{i}}+\varepsilon \nabla u_{i 0 \varepsilon}^{(k)}\right|^{2} \mathrm{~d} x-\frac{1}{2} \int_{\Omega}\left|\nabla\left(u_{i \varepsilon}^{(k)}\left(x, T_{1}\right)\right)^{m_{i}}+\varepsilon \nabla u_{i \varepsilon}^{(k)}\left(x, T_{1}\right)\right|^{2} \mathrm{~d} x \\
& +\frac{1}{4} \iint_{\mathrm{Q}_{1}} f_{i \varepsilon}^{2}\left(x, t, u_{1 \varepsilon}^{(k-1)}, u_{2 \varepsilon}^{(k-1)}\right) \mathrm{d} x \mathrm{~d} t+\frac{m_{i}}{2} \iint_{\mathrm{Q}_{1}} f_{i \varepsilon}^{2}\left(x, t, u_{1 \varepsilon}^{(k-1)}, u_{2 \varepsilon}^{(k-1)}\right)\left|u_{i \varepsilon}^{(k)}\right|^{m_{i}-1} \mathrm{~d} x \mathrm{~d} t \\
& +\frac{2 m_{i}}{\left(m_{i}+1\right)^{2}} \iint_{\mathrm{Q}_{1}}\left|\frac{\partial}{\partial t}\left(u_{i \varepsilon}^{(k)}\right)^{\left(m_{i}+1\right) / 2}\right|^{2} \mathrm{~d} x \mathrm{~d} t+\varepsilon^{2} \iint_{\mathrm{Q}_{1}}\left|\frac{\partial u_{i \varepsilon}^{(k)}}{\partial t}\right|^{2} \mathrm{~d} x \mathrm{~d} t .
\end{aligned}
$$

Noticing that the first term of the left side of the above inequality can be rewritten as

$$
\iint_{Q_{T_{1}}} \frac{\partial u_{i \varepsilon}^{(k)}}{\partial t} \frac{\partial\left(u_{i \varepsilon}^{(k)}\right)^{m_{i}}}{\partial t} \mathrm{~d} x \mathrm{~d} t=\frac{4 m_{i}}{\left(m_{i}+1\right)^{2}} \iint_{Q_{T_{1}}}\left|\frac{\partial}{\partial t}\left(u_{i \varepsilon}^{(k)}\right)^{\left(m_{i}+1\right) / 2}\right|^{2} \mathrm{~d} x \mathrm{~d} t .
$$

Then we have

$$
\begin{gathered}
\frac{2 m_{i}}{\left(m_{i}+1\right)^{2}} \iint_{Q_{T_{1}}}\left|\frac{\partial}{\partial t}\left(u_{i \varepsilon}^{(k)}\right)^{\left(m_{i}+1\right) / 2}\right|^{2} \mathrm{~d} x \mathrm{~d} t+\left(\varepsilon-\varepsilon^{2}\right) \iint_{Q_{T_{1}}}\left|\frac{\partial u_{i \varepsilon}^{(k)}}{\partial t}\right|^{2} \mathrm{~d} x \mathrm{~d} t \\
\leq \frac{1}{2} \int_{\Omega}\left|\nabla\left(u_{i 0 \varepsilon}^{(k)}\right)^{m_{i}}+\varepsilon \nabla u_{i 0 \varepsilon}^{(k)}\right|^{2} \mathrm{~d} x+\frac{1}{4} \iint_{Q_{T_{1}}} f_{i \varepsilon}^{2}\left(x, t, u_{1 \varepsilon}^{(k-1)}, u_{2 \varepsilon}^{(k-1)}\right) \mathrm{d} x \mathrm{~d} t \\
+\frac{m_{i}}{2} \iint_{Q_{T_{1}}} f_{i \varepsilon}^{2}\left(x, t, u_{1 \varepsilon}^{(k-1)}, u_{2 \varepsilon}^{(k-1)}\right)\left|u_{i \varepsilon}^{(k)}\right|^{m_{i}-1} \mathrm{~d} x \mathrm{~d} t .
\end{gathered}
$$

Therefore

$$
\iint_{Q_{T_{1}}}\left|\frac{\partial}{\partial t}\left(u_{i \varepsilon}^{(k)}\right)^{\left(m_{i}+1\right) / 2}\right|^{2} \mathrm{~d} x \mathrm{~d} t \leq C
$$


Furthermore, we can obtain

$$
\begin{aligned}
& \iint_{Q_{T_{1}}}\left|\frac{\partial}{\partial t}\left(u_{i \varepsilon}^{(k)}\right)^{m_{i}}\right|^{2} \mathrm{~d} x \mathrm{~d} t=\frac{4 m_{i}}{\left(m_{i}+1\right)^{2}} \iint_{\mathrm{Q}_{T_{1}}}\left(u_{i \varepsilon}^{(k)}\right)^{m_{i}-1}\left|\frac{\partial}{\partial t}\left(u_{i \varepsilon}^{(k)}\right)^{\left(m_{i}+1\right) / 2}\right|^{2} \leq C, \\
& \iint_{Q_{T_{1}}}\left|\frac{\partial}{\partial t} u_{i \varepsilon}^{(k)}\right|^{2} \mathrm{~d} x \mathrm{~d} t \leq C .
\end{aligned}
$$

Following (2.8), (2.9), (2.12) and the uniqueness of the weak limits, it is easy to know that, as $k \rightarrow \infty$,

$$
\begin{aligned}
& u_{i \varepsilon}^{(k)} \rightarrow u_{i \varepsilon}, f_{i \varepsilon}\left(x, t, u_{1 \varepsilon}^{(k)}, u_{2 \varepsilon}^{(k)}\right) \rightarrow f_{i \varepsilon}\left(x, t, u_{1 \varepsilon}, u_{2 \varepsilon}\right), \quad \text { a.e. in } Q_{T_{1}} \\
& \frac{\partial u_{i \varepsilon}^{(k)}}{\partial t} \rightarrow \frac{\partial u_{i \varepsilon}}{\partial t}, \frac{\partial\left(u_{i \varepsilon}^{(k)}\right)^{m_{i}}}{\partial t} \rightarrow \frac{\partial u_{i \varepsilon}^{m_{i}}}{\partial t}, \quad \text { in } \quad L^{2}\left(Q_{T_{1}}\right),
\end{aligned}
$$

where $\rightarrow$ stands for weak convergence, $i=1$, 2. Furthermore (2.11) implies that there exists $v_{s} \in L^{2}\left(Q_{T_{1}}\right), s=1, \ldots, n$, such that

$$
\frac{\partial\left(\left(u_{i \varepsilon}^{(k)}\right)^{m_{i}}+\varepsilon u_{i \varepsilon}^{(k)}\right)}{\partial x_{s}} \rightarrow v_{s} \text { a.e. in } L^{2}\left(Q_{T_{1}}\right) .
$$

Hence,

$$
\iint_{Q_{T_{1}}}-u_{i \varepsilon} \frac{\partial \varphi_{i}}{\partial t}+v \nabla \varphi_{i} \mathrm{~d} x \mathrm{~d} t-\int_{\Omega} u_{i 0 \varepsilon}(x) \varphi_{i}(x, 0) \mathrm{d} x=\iint_{Q_{T_{1}}} f_{i}\left(x, t, u_{1 \varepsilon}, u_{2 \varepsilon}\right) \varphi_{i} \mathrm{~d} x \mathrm{~d} t,(2
$$

where $v=\left(v_{1}, \ldots, v_{n}\right), \varphi_{i} \in C^{2}\left(\bar{Q}_{T_{1}}\right)$ with $\left.\varphi_{i}\right|_{\partial \Omega \times\left(0, T_{1}\right)}=0, \phi_{i}\left(x, T_{1}\right)=0, i=1,2$.

Now for any $\phi_{i}$ given as before, we show

$$
\iint_{Q_{T_{1}}} \nabla\left(\left(u_{i \varepsilon}^{(k)}\right)^{m_{i}}+\varepsilon u_{i \varepsilon}^{(k)}\right) \nabla \varphi_{i} \mathrm{~d} x \mathrm{~d} t=\iint_{Q_{T_{1}}} v \nabla \varphi_{i} \mathrm{~d} x \mathrm{~d} t, \quad \text { as } k \rightarrow \infty .
$$

For any $w \in L^{2}\left(0, T_{1} ; H_{0}^{1}(\Omega)\right), \zeta \in C^{1}\left(\bar{Q}_{T_{1}}\right), 0 \leq \zeta \leq 1,\left.\zeta\right|_{\partial \Omega \times\left(0, T_{1}\right)}=0$ with $\zeta\left(x, T_{1}\right)=$ 0 , multiplying (2.4) by $\zeta\left(\left(u_{i \varepsilon}^{(k)}\right)^{m_{i}}+\varepsilon u_{i \varepsilon}^{(k)}\right)$ and integrating over $Q_{T_{1}}$, we have

$$
\begin{aligned}
& \iint_{Q_{T_{1}}} \zeta \mid \nabla\left.\left(\left(u_{i \varepsilon}^{(k)}\right)^{m_{i}}+\varepsilon u_{i \varepsilon}^{(k)}\right)\right|^{2} \mathrm{~d} x \mathrm{~d} t \\
&=\iint_{Q_{T_{1}}} \zeta\left(\left(u_{i \varepsilon}^{(k)}\right)^{m_{i}}+\varepsilon u_{i \varepsilon}^{(k)}\right) f_{i}\left(x, t, u_{1 \varepsilon}^{(k-1)}, u_{2 \varepsilon}^{(k-1)}\right) \mathrm{d} x \mathrm{~d} t \\
& \quad+\int_{\Omega} \zeta(x, 0)\left(\frac{1}{m_{i}+1}\left(u_{i 0 \varepsilon}^{(k)}\right)^{m_{i}+1}+\frac{\varepsilon}{2}\left(u_{i 0 \varepsilon}^{(k)}\right)^{2}\right) \mathrm{d} x \\
& \quad+\iint_{Q_{T_{1}}}\left(\frac{1}{m_{i}+1}\left(u_{i \varepsilon}^{(k)}\right)^{m_{i}+1}+\frac{\varepsilon}{2}\left(u_{i \varepsilon}^{(k)}\right)^{2}\right) \zeta_{t} \mathrm{~d} x \mathrm{~d} t \\
& \quad-\iint_{\mathrm{Q}_{T_{1}}}\left(\left(u_{i \varepsilon}^{(k)}\right)^{m_{i}}+\varepsilon u_{i \varepsilon}^{(k)}\right) \nabla\left(\left(u_{i \varepsilon}^{(k)}\right)^{m_{i}}+\varepsilon u_{i \varepsilon}^{(k)}\right) \nabla \zeta \mathrm{d} x \mathrm{~d} t .
\end{aligned}
$$


Notice that

$$
\begin{aligned}
& \iint_{\mathrm{Q}_{T_{1}}} \zeta\left|\nabla\left(\left(u_{i \varepsilon}^{(k)}\right)^{m_{i}}+\varepsilon u_{i \varepsilon}^{(k)}\right)\right|^{2} \mathrm{~d} x \mathrm{~d} t \\
& \quad-\iint_{\mathrm{Q}_{T_{1}}} \zeta \nabla\left(\left(u_{i \varepsilon}^{(k)}\right)^{m_{i}}+\varepsilon u_{i \varepsilon}^{(k)}\right) \nabla w \mathrm{~d} x \mathrm{~d} t-\iint_{\mathrm{Q}_{T_{1}}} \zeta \nabla w \nabla\left(\left(u_{i \varepsilon}^{(k)}\right)^{m_{i}}+\varepsilon u_{i \varepsilon}^{(k)}-w\right) \mathrm{d} x \mathrm{~d} t \\
& \quad=\iint_{Q_{T_{1}}} \zeta \nabla\left(\left(u_{i \varepsilon}^{(k)}\right)^{m_{i}}+\varepsilon u_{i \varepsilon}^{(k)}-w\right) \nabla\left(\left(u_{i \varepsilon}^{(k)}\right)^{m_{i}}+\varepsilon u_{i \varepsilon}^{(k)}-w\right) \mathrm{d} x \mathrm{~d} t \geq 0
\end{aligned}
$$

from (2.17), we get

$$
\begin{aligned}
\iint_{Q_{T_{1}}} \zeta\left(\left(u_{i \varepsilon}^{(k)}\right)^{m_{i}}+\varepsilon u_{i \varepsilon}^{(k)}\right) f_{i}\left(x, t, u_{1 \varepsilon}^{(k-1)}, u_{2 \varepsilon}^{(k-1)}\right) \mathrm{d} x \mathrm{~d} t \\
\quad+\int_{\Omega} \zeta(x, 0)\left(\frac{1}{m_{i}+1}\left(u_{i 0 \varepsilon}^{(k)}\right)^{m_{i}+1}+\frac{\varepsilon}{2}\left(u_{i 0 \varepsilon}^{(k)}\right)^{2}\right) \mathrm{d} x \\
\quad+\iint_{Q_{T_{1}}}\left(\frac{1}{m_{i}+1}\left(u_{i \varepsilon}^{(k)}\right)^{m_{i}+1}+\frac{1}{2}\left(u_{i \varepsilon}^{(k)}\right)^{2}\right) \zeta t \mathrm{~d} x \mathrm{~d} t \\
\quad-\iint_{Q_{T_{1}}}\left(\left(u_{i \varepsilon}^{(k)}\right)^{m_{i}}+\varepsilon u_{i \varepsilon}^{(k)}\right) \nabla\left(\left(u_{i \varepsilon}^{(k)}\right)^{m_{i}}+\varepsilon u_{i \varepsilon}^{(k)}\right) \nabla \zeta \mathrm{d} x \mathrm{~d} t \\
\left.\quad-\iint_{Q_{T_{1}}} \zeta \nabla\left(\left(u_{i \varepsilon}^{(k)}\right)^{m_{i}}+\varepsilon u_{i \varepsilon}^{(k)}\right)\right) \nabla w \mathrm{~d} x \mathrm{~d} t-\iint_{Q_{T_{1}}} \zeta \nabla w \nabla\left(\left(u_{i \varepsilon}^{(k)}\right)^{m_{i}}+\varepsilon u_{i \varepsilon}^{(k)}-w\right) \mathrm{d} x \mathrm{~d} t \geq 0 .
\end{aligned}
$$

Letting $k \rightarrow \infty$, then

$$
\begin{aligned}
& \iint_{Q_{T_{1}}} \zeta\left(\left(u_{i \varepsilon}\right)^{m_{i}}+\varepsilon u_{i \varepsilon}\right) f_{i}\left(x, t, u_{1 \varepsilon}, u_{2 \varepsilon}\right) \varphi_{i} \mathrm{~d} x \mathrm{~d} t \\
& \quad+\int_{\Omega} \zeta(x, 0)\left(\frac{u_{i 0 \varepsilon}^{m_{i}+1}}{m_{i}+1}+\frac{\varepsilon}{2} u_{i 0 \varepsilon}^{2}\right) \mathrm{d} x \\
& \quad+\iint_{\mathrm{Q}_{T_{1}}}\left(\frac{u_{i \varepsilon}^{m_{i}+1}}{m_{i}+1}+\frac{1}{2} u_{i \varepsilon}^{2}\right) \zeta \mathrm{d} x \mathrm{~d} t-\iint_{\mathrm{Q}_{T_{1}}}\left(u_{i \varepsilon}^{m_{i}}+\varepsilon u_{i \varepsilon}\right) \nu \nabla \zeta \mathrm{d} x \mathrm{~d} t \\
& \quad-\iint_{Q_{T_{1}}} \zeta \nu \nabla w \mathrm{~d} x \mathrm{~d} t-\iint_{Q_{T_{1}}} \zeta \nabla w \nabla\left(u_{i \varepsilon}^{m_{i}}+\varepsilon u_{i \varepsilon}-w\right) \mathrm{d} x \mathrm{~d} t \geq 0 .
\end{aligned}
$$

Set $\varphi_{i}=\zeta\left(u_{i \varepsilon}^{m_{i}}+\varepsilon u_{i \varepsilon}\right)$ in (2.15), we obtain

$$
\begin{aligned}
& \iint_{Q_{T_{1}}} \zeta\left(u_{i \varepsilon}^{m_{i}}+\varepsilon u_{i \varepsilon}\right) f_{i}\left(x, t, u_{1 \varepsilon}, u_{2 \varepsilon}\right) \mathrm{d} x \mathrm{~d} t \\
& \quad+\int_{\Omega} \zeta(x, 0)\left(\frac{u_{i 0 \varepsilon}^{m_{i}+1}}{m_{i}+1}+\frac{\varepsilon}{2} u_{i 0 \varepsilon}^{2}\right) \mathrm{d} x+\iint_{\mathrm{Q}_{T_{1}}}\left(\frac{u_{i \varepsilon}^{m_{i}+1}}{m_{i}+1}+\frac{u_{i \varepsilon}^{2}}{2}\right) \zeta_{t} \mathrm{~d} x \mathrm{~d} t \\
& \quad=\iint_{Q_{T_{1}}}\left(u_{i \varepsilon}^{m_{i}}+\varepsilon u_{i \varepsilon}\right) v \nabla \zeta \mathrm{d} x \mathrm{~d} t+\iint_{Q_{T_{1}}} \zeta v \nabla\left(u_{i \varepsilon}^{m_{i}}+\varepsilon u_{i \varepsilon}\right) \mathrm{d} x \mathrm{~d} t .
\end{aligned}
$$


Substituting the above equation into (2.18), we get

$$
\iint_{Q_{T_{1}}} \zeta(v-\nabla w) \nabla\left(u_{i \varepsilon}^{m_{i}}+\varepsilon u_{i \varepsilon}-w\right) \mathrm{d} x \mathrm{~d} t \geq 0 .
$$

Taking $w=u_{i \varepsilon}^{m_{i}}+\varepsilon u_{i \varepsilon}-\delta \varphi_{i}, \delta \geq 0$ in (2.19) and then let $\delta \rightarrow 0$, we obtain

$$
\iint_{Q_{T_{1}}} \zeta\left(v-\nabla\left(u_{i \varepsilon}^{m_{i}}+\varepsilon u_{i \varepsilon}\right)\right) \nabla \varphi_{i} \mathrm{~d} x \mathrm{~d} t \geq 0,
$$

where $\varphi_{i} \in C^{1}\left(\bar{Q}_{T_{1}}\right)$ with $\left.\varphi_{i}\right|_{\partial \Omega \times\left(0, T_{1}\right)}=0$. Obviously, if we let $\delta \leq 0$, we can get the inverted inequality. So we can obtain (2.16) by choosing suitable $\zeta$, s.t. $\operatorname{supp} \phi_{i} \subset \operatorname{supp} \zeta$ and $\zeta=1$ on $\operatorname{supp} \phi_{i}$.

In summary, we have proved that $u_{\varepsilon}=\left(u_{1 \varepsilon}, u_{2 \varepsilon}\right)$ is a weak solution of (2.1)-(2.3).

Now, we will prove that the limit of $u_{\varepsilon}=\left(u_{1 \varepsilon}, u_{2 \varepsilon}\right)$ is a weak solution of (1.1)-(1.3). Since $u_{\varepsilon}=\left(u_{1 \varepsilon}, u_{2 \varepsilon}\right)$ satisfies similar estimates as (2.10)-(2.12), combining the property of $f_{i \varepsilon}$, we know that there are functions $u_{i}^{m_{i}} \in L^{2}\left(0, T_{1} ; H_{0}^{1}(\Omega)\right), u_{i t}, u_{i t}^{m_{i}} \in L^{2}\left(Q_{T_{1}}\right), i=1$, 2 , such that for some subsequence of $\left(u_{1 \varepsilon}, u_{2 \varepsilon}\right)$, denoted by itself for simplicity, when $\varepsilon$ $\rightarrow 0$

$$
\begin{aligned}
& u_{i \varepsilon} \rightarrow u_{i}, f_{i \varepsilon}\left(x, t, u_{1 \varepsilon}, u_{2 \varepsilon}\right) \rightarrow f_{i}\left(x, t, u_{1}, u_{2}\right), \quad \text { a.e. in } Q_{T_{1}}, \\
& \frac{\partial u_{i \varepsilon}}{\partial t} \rightarrow \frac{\partial u_{i}}{\partial t}, \frac{\partial u_{i \varepsilon}^{m_{i}}}{\partial t} \rightarrow \frac{\partial u_{i}^{m_{i}}}{\partial t}, \quad \text { in } \quad L^{2}\left(Q_{T_{1}}\right) .
\end{aligned}
$$

Then a similar argument as above shows that $u=\left(u_{1}, u_{2}\right)$ is a weak solution of (1.1)(1.3).

The following is the uniqueness result to the solution of the system.

Theorem 2.2. Assume that $f=\left(f_{1}, f_{2}\right)$ is Lipschitz continuous in $\left(u_{1}, u_{2}\right)$, then (1.1)(1.3) has a unique solution.

Proof. Assume that $u=\left(u_{1}, u_{2}\right), v=\left(v_{1}, v_{2}\right)$ are two solutions of (1.1)-(1.3). Form Definition 1, we see that

$$
\begin{gathered}
\int_{0}^{t} \int_{\Omega}-u_{i} \frac{\partial \varphi_{i}}{\partial t}+\nabla u_{i}^{m_{i}} \nabla \varphi_{i} \mathrm{~d} x \mathrm{~d} t+\int_{\Omega} u_{i}(x, t) \varphi_{i}(x, t) \mathrm{d} x-\int_{\Omega} u_{i 0}(x) \varphi_{i}(x, 0) \mathrm{d} x \\
=\int_{0}^{t} \int_{\Omega} f_{i}\left(x, t, u_{1}, u_{2}\right) \varphi_{i} \mathrm{~d} x \mathrm{~d} t, \quad \text { a.e. } t \in(0, T) . \\
\int_{0}^{t} \int_{\Omega}-v_{i} \frac{\partial \varphi_{i}}{\partial t}+\nabla v_{i}^{m_{i}} \nabla \varphi_{i} \mathrm{~d} x \mathrm{~d} t+\int_{\Omega} v_{i}(x, t) \varphi_{i}(x, t) \mathrm{d} x-\int_{\Omega} v_{i 0}(x) \varphi_{i}(x, 0) \mathrm{d} x \\
=\int_{0}^{t} \int_{\Omega} f_{i}\left(x, t, v_{1}, v_{2}\right) \varphi_{i} \mathrm{~d} x \mathrm{~d} t, \quad \text { a.e. } t \in(0, T) .
\end{gathered}
$$


Subtracting the two equations, we get

$$
\begin{aligned}
& \int_{\Omega}\left(u_{i}(x, t)-v_{i}(x, t)\right) \varphi_{i}(x, t) \mathrm{d} x \\
& \quad=\int_{0}^{t} \int_{\Omega}\left(u_{i}-v_{i}\right)\left(\varphi_{i t}+\Phi(x, s) \Delta \varphi_{i}\right) \mathrm{d} x \mathrm{~d} s+\int_{0}^{t} \int_{\Omega}\left(f_{i}\left(x, t, u_{1}, u_{2}\right)-f_{i}\left(x, t, v_{1}, v_{2}\right)\right) \varphi_{i} \mathrm{~d} x \mathrm{~d} s,
\end{aligned}
$$

where

$$
\Phi(x, s) \equiv \int_{0}^{1} m_{i}\left(\theta u_{i}+(1-\theta) v_{i}\right)^{m_{1}-1} \mathrm{~d} \theta .
$$

Since $\left(u_{1}, u_{2}\right)$ and $\left(v_{1}, v_{2}\right)$ are bounded on $Q_{t}$, it follows from $m>1, \Phi(x, s)$ is a bounded nonnegative function. Thus, appropriate test function $\phi_{i}$ may be chosen exactly as in [[17], pp. 118-123] and combined with the Lipschitz continuity of $f_{i}$ to obtain

$$
\int_{\Omega}\left|u_{i}(x, t)-v_{i}(x, t)\right| \mathrm{d} x \leq C \int_{0}^{t} \int_{\Omega}\left|u_{1}-v_{1}\right|+\left|u_{2}-v_{2}\right| \mathrm{d} x \mathrm{~d} s, \quad i=1,2 .
$$

where $C>0$ is a bounded constant. Further, we have

$$
\int_{\Omega}\left|u_{1}(x, t)-v_{1}(x, t)\right|+\left|u_{2}(x, t)-v_{2}(x, t)\right| \mathrm{d} x \leq C \int_{0}^{t} \int_{\Omega}\left|u_{1}-v_{1}\right|+\left|u_{2}-v_{2}\right| \mathrm{d} x \mathrm{~d} s .
$$

Combined with the Gronwall's lemma, we see that $u_{i} \equiv v_{i}, i=1$, 2. The proof is completed.

\section{Acknowledgements}

The authors express their deep thanks to the referees for their very helpful suggestions to improve some results in this paper. This work is supported by "the Fundamental Research Funds for the Central Universities" (Grant No. HIT. NSRIF. 2011006) and also by the 985 project of Harbin Institute of Technology.

\section{Authors' contributions}

DZ and JS carried out the proof of existence, BW conceived of the study, and participated in its design and coordination. All authors read and approved the final manuscript.

\section{Competing interests}

The authors declare that they have no competing interests.

Received: 30 November 2010 Accepted: 16 June 2011 Published: 16 June 2011

\section{References}

1. Arossox, DG, Craxdall, MG, Peletier, LA: Stabilization of solutions of a degenerate nonlinear diffusion problem. Nonlinear Anal. 6(10), 1001-1022 (1982). doi:10.1016/0362-546X(82)90072-4

2. Bear, J: Dynamics of Fluids in Porous Media. Elsevier, New York (1972)

3. Lei, PD, Zheng, SN: Global and nonglobal weak solutions to a degenerate parabolic system. J Math Anal Appl. 324(1), 177-198 (2006). doi:10.1016/j.jmaa.2005.12.012

4. Okubo, A: Diffusion and Ecological Problems: Mathematical Models. In Biomathematics, vol. 10,Springer, Berlin, Heidelberg, New York (1980)

5. Meinhardt, H: Models of Biological Pattern Formation. Academic Press, London (1982)

6. Romanvoskii, YM, Stepanova, NV, Chernavskii, DS: Mathematical biophysics. Nauka, Moscow (1984). (in Russian)

7. Constantin, A, Escher, J, Yin, Z: Global solutions for quasilinear parabolic system. J Differ Equ. 197(1), 73-84 (2004). doi:10.1016/50022-0396(03)00165-7

8. Dickstein, F, Escobedo, M: A maximum principle for semilinear parabolic systems and application. Nonlinear Anal. 45(7), 825-837 (2001). doi:10.1016/50362-546X(99)00419-8

9. Pierre, M, Schmidt, D: Blowup in reaction diffusion systems with dissipation of mass. SIAM J Math Anal. 28(2), 259-269 (1997). doi:10.1137/S0036141095295437 
10. Wang, MX, Wei, YF: Blow-up properties for a degenerate parabolic system with nonlinear localized sources. J Math Anal Appl. 343(2), 621-635 (2008). doi:10.1016/j.jmaa.2008.01.073

11. Cui, ZJ, Yang, ZD: Boundedness of global solutions for a nonlinear degenerate parabolic (porous medium) system with localized sources. Appl Math Comput. 198(2), 882-895 (2008). doi:10.1016/j.amc.2007.09.037

12. Litcanu, G, Morales-Rodrigo, C: Global solutions and asymptotic behavior for a parabolic degenerate coupled system arising from biology. Nonlinear Anal. 72(1), 77-98 (2008)

13. Le, D: Higher integrability for gradients of solutions to degenerate parabolic systems. Discr Contin Dyn Syst. 26(2) 597-608 (2010)

14. Ladyzenskaja, OA, Solonnikov, VA, Ural'ceva, NN: Linear and quasilinear equations of parabolic type. Translation of Mathematical Monographs. American Mathematical Society, Providence. 23 (1968)

15. Friedman, A: Partial Differential Equations of Parabolic Type. Prentice-Hall Inc, Engle-wood Cliffs (1964)

16. Coddington, E, Levinson, N: Theory of Ordinary Differential Equations. McGraw-Hill, New York (1955)

17. Anderson, JR: Local existence and uniqueness of solutions of degenerate parabolic equations. Commun Partial Differ Equ. 16, 105-143 (1991). doi:10.1080/03605309108820753

doi:10.1186/1687-1847-2011-12

Cite this article as: Zhang et al.: Local existence and uniqueness of solutions of a degenerate parabolic system. Advances in Difference Equations 2011 2011:12.

\section{Submit your manuscript to a SpringerOpen ${ }^{\circ}$} journal and benefit from:

- Convenient online submission

- Rigorous peer review

- Immediate publication on acceptance

- Open access: articles freely available online

- High visibility within the field

- Retaining the copyright to your article

Submit your next manuscript at $\gg$ springeropen.com 